\title{
Existence and Uniqueness for the Boundary Value Problems of Nonlinear Fractional Differential Equation
}

\author{
Yufeng Sun ${ }^{1}$, Zheng Zeng², Jie Song ${ }^{*}$ \\ ${ }^{1}$ School of Mathematics and Statistics, Shaoguan University, Shaoguan, China \\ ${ }^{2}$ Office of Party Committee, Foshan University, Foshan, China \\ Email: suny2001@sina.com,zz@fosu.edu.cn, *jiesong2004@163.com
}

How to cite this paper: Sun, Y.F., Zeng, Z. and Song, J. (2017) Existence and Uniqueness for the Boundary Value Problems of Nonlinear Fractional Differential Equation. Applied Mathematics, 8, 312-323. https://doi.org/10.4236/am.2017.83026

Received: October 6, 2016

Accepted: March 21, 2017

Published: March 24, 2017

Copyright (c) 2017 by authors and Scientific Research Publishing Inc. This work is licensed under the Creative Commons Attribution International License (CC BY 4.0).

http://creativecommons.org/licenses/by/4.0/

\section{c) (†) Open Access}

\begin{abstract}
This paper studies the existence and uniqueness of solutions for a class of boundary value problems of nonlinear fractional order differential equations involving the Caputo fractional derivative by employing the Banach's contraction principle and the Schauder's fixed point theorem. In addition, an example is given to demonstrate the application of our main results.
\end{abstract}

\section{Keywords}

Fractional Order Differential Equations, Boundary Value Problem, Caputo Fractional Derivative, Fractional Integral, Fixed Point

\section{Introduction}

This paper considers the following boundary value problems of fractional order differential equations

$$
\left\{\begin{array}{l}
{ }^{c} D_{a}^{\alpha} x(t)=f(t, x(t)), \text { for } \quad t \in J=[a, b], \quad n-1<\alpha \leq n, \\
x^{(k)}(a)=x_{k}, \quad k=0,1,2, \cdots, n-2 ; \quad x^{(n-1)}(b)=x_{b}
\end{array}\right.
$$

where ${ }^{c} D_{a}^{\alpha}$ is the Caputo fractional derivative, $f: J \times R \rightarrow R$ is continuous function and $x_{0}, x_{1}, \cdots, x_{n-2}, x_{b}$ are real constants.

Fractional order Differential equations have recently proved to be valuable tools in the modeling of many phenomena in various fields of science and engineering. Applications can be found in fields of control, porous media, eletromagnetic, etc. (see [1] [2] [3] [4] [5]). There has been a significant progress in the investigation of fractional differential equations in recent years, The readers are referred to the monographs of Oldham and Spanier [1], Miller and Ross [2], 
Podlubny [3], Hilfer [5] and the papers of Agarwal et al. [6], El-Sayed [7] [8] [9] [10], Benchohra et al. [11] [12], Yu and Gao [13] [14], Zhang [15], He [4] and the others references therein [16]-[23].

Recently some basic theory for the initial value problems of fractional differential equations involving Riemann-Liouville differential operator $(0<\alpha<1)$ has been discussed by Lakshmikantham et al. [24] [25] [26]. In a series of papers (see [6] [11]), the authors considered some classes of boundary value problems for differential equations involving Riemann-Liouville and Caputo fractional derivatives of order $0<\alpha<1$ and $2<\alpha<3$.

This paper generalizes the results of the papers above [6] and presents some existence theorems for the boundary value problems (BVP) (1.1). Two theorems are based on the Banach fixed point theorem, and the others are based on Schauder's fixed point theorem and Leray-Schauder type nonlinear alternative. An example is given to demonstrate the application of our main results.

\section{Preliminaries}

Some notions and Lemmas are important in order to state our results. Denote by $C(J, R)$ the Banach space of all continuous functions from $J$ into $R$ with the norm

$$
\|x\|_{\infty}:=\sup _{t \in J}\{|x(t)|\}, \quad J=[a, b] .
$$

Definition 2.1 ([6] [11]) The fractional order integral of the function $h(t) \in L^{1}\left([a, b], R_{+}\right)$is defined by

$$
I_{a}^{\alpha} h(t)=\frac{1}{\Gamma(\alpha)} \int_{a}^{t}(t-s)^{\alpha-1} h(s) \mathrm{d} s
$$

where $\Gamma$ is the gamma function.

Definition 2.2 ([6] [11]) For a function $h$ given on the interval [a,b], the $\alpha$ th Caputo fractional-order derivative of $h$ is defined by

$$
\left({ }^{c} D_{a}^{\alpha} h\right)(t)=\frac{1}{\Gamma(n-\alpha)} \int_{a}^{t}(t-s)^{n-\alpha-1} h^{(n)}(s) \mathrm{d} s
$$

where $n=[\alpha]+1$ and $[\alpha]$ denotes the integer part of $\alpha$.

A solution of the problem (1.1) is defined as follows.

Definition 2.3 A function $x(t) \in C^{n-1}(J, R)$ that satisfies (1.1) is called a solution of (1.1).

Lemma 2.1 ([15]) Let $\alpha>0$, then the differential equation

$$
{ }^{c} D_{a}^{\alpha} h(t)=0
$$

has solutions

$$
\begin{aligned}
& h(t)=c_{0}+c_{1}(t-a)+c_{2}(t-a)^{2}+\cdots+c_{n-1}(t-a)^{n-1}, \\
& c_{i} \in R, i=0,1,2, \cdots, n-1, n=[\alpha]+1 .
\end{aligned}
$$

Lemma 2.2 Let $\alpha>0$, then

$$
I_{a}^{\alpha c} D_{a}^{\alpha} h(t)=h(t)+c_{0}+c_{1}(t-a)+c_{2}(t-a)^{2}+\cdots+c_{n-1}(t-a)^{n-1} .
$$


In particular, when $a=0$,

$$
I^{\alpha c} D^{\alpha} h(t)=h(t)+c_{0}+c_{1} t+c_{2} t^{2}+\cdots+c_{n-1} t^{n-1},
$$

for some $c_{i} \in R, i=0,1,2, \cdots, n-1, n=[\alpha]+1$.

Proof. By (2.1), (2.2),

$$
\begin{aligned}
& I_{a}^{\alpha} D_{a}^{\alpha} h(t)=\frac{1}{\Gamma(\alpha)} \int_{a}^{t}(t-s)^{\alpha-1} D_{a}^{\alpha} h(s) \mathrm{d} s \\
& =\frac{1}{\Gamma(\alpha)} \int_{a}^{t}(t-s)^{\alpha-1}\left[\frac{1}{\Gamma(n-\alpha)} \int_{a}^{s}(s-\tau)^{n-\alpha-1} h^{(n)}(\tau) \mathrm{d} \tau\right] \mathrm{d} s \\
& =\frac{1}{\Gamma(\alpha) \Gamma(n-\alpha)} \int_{a}^{t} h^{(n)}(\tau) \mathrm{d} \tau \int_{\tau}^{t}(t-s)^{\alpha-1}(s-\tau)^{n-\alpha-1} \mathrm{~d} s \\
& =\frac{1}{\Gamma(\alpha) \Gamma(n-\alpha)} \int_{a}^{t} h^{(n)}(\tau) \mathrm{d} \tau \frac{(\alpha-1)}{(n-\alpha)} \int_{\tau}^{t}(t-s)^{\alpha-2}(s-\tau)^{n-\alpha} \mathrm{d} s \\
& =\frac{1}{\Gamma(\alpha) \Gamma(n-\alpha)} \int_{a}^{t} h^{(n)}(\tau) \mathrm{d} \tau \frac{(\alpha-1)(\alpha-2) \cdots 2 \times 1}{(n-\alpha)(n-\alpha+1) \cdots(n-2)} \int_{\tau}^{t}(s-\tau)^{n-2} \mathrm{~d} s \\
& =\frac{1}{\Gamma(n)} \int_{a}^{t}(t-\tau)^{n-1} h^{(n)}(\tau) \mathrm{d} \tau \\
& =\frac{1}{\Gamma(n)} \int_{a}^{t}(t-\tau)^{n-1} \mathrm{~d} h^{(n-1)}(\tau) \\
& =-\frac{h^{(n-1)}(a)}{\Gamma(n)}(t-a)^{n-1}-\frac{h^{(n-2)}(a)}{\Gamma(n-1)}(t-a)^{n-2}+\frac{1}{\Gamma(n-2)} \int_{a}^{t}(t-\tau)^{n-3} h^{(n-2)}(\tau) \mathrm{d} \tau \\
& =-\frac{h^{(n-1)}(a)}{\Gamma(n)}(t-a)^{n-1}-\frac{h^{(n-2)}(a)}{\Gamma(n-1)}(t-a)^{n-2}-\cdots-\frac{h^{\prime}(a)}{\Gamma(2)}(t-a)-\frac{h(a)}{\Gamma(1)}+h(t) \\
& =h(t)+c_{0}+c_{1}(t-a)+c_{2}(t-a)^{2}+\cdots+c_{n-1}(t-a)^{n-1}
\end{aligned}
$$

Lemma 2.3 ([27]) The relation

$$
{ }^{c} D_{a}^{\alpha} I_{a}^{\alpha} h(t)=h(t), I_{a}^{\alpha} I_{a}^{\beta} h(t)=I_{a}^{\alpha+\beta} h(t)
$$

is valid in following case

$$
\text { Re } \alpha>0, \quad \operatorname{Re} \beta>0, \quad h(t) \in L^{1}(a, b) .
$$

As a consequence of Lemmas 2.1, Lemmas 2.2 and Lemmas 2.3, the following result is useful in what follows.

Lemma 2.4 Let $n-1<\alpha<n, n=[\alpha]+1$, and let $h: J \rightarrow R$ be continuous. A function $x(t)$ is a solution of the fractional BVP

$$
\left\{\begin{array}{l}
{ }^{c} D_{a}^{\alpha} x(t)=h(t), \quad t \in J, \\
x^{(k)}(a)=x_{k}, \quad k=0,1,2, \cdots, n-2 ; \quad x^{(n-1)}(b)=x_{b},
\end{array}\right.
$$

if and only if $x(t)$ is a solution of the fractional integral equation 


$$
\begin{aligned}
x(t)= & \frac{1}{\Gamma(\alpha)} \int_{a}^{t}(t-s)^{\alpha-1} h(s) \mathrm{d} s+\left(\frac{x_{b}}{(n-1) !}+\frac{h(a)(b-a)^{\alpha-n+1}}{(n-2) ! \Gamma(\alpha-n+2)}\right)(t-a)^{n-1} \\
& -\frac{(t-a)^{n-1}}{(n-1) ! \Gamma(\alpha-n+1)} \int_{a}^{b}(b-s)^{\alpha-n} h(s) \mathrm{d} s+\sum_{k=0}^{n-2} \frac{x_{k}}{k !}(t-a)^{k} .
\end{aligned}
$$

Proof. Assume $x(t)$ satisfies (2.4), then Lemma 2.2 implies that $x(t)=c_{0}+c_{1}(t-a)+c_{2}(t-a)^{2}+\cdots+c_{n-1}(t-a)^{n-1}+\frac{1}{\Gamma(\alpha)} \int_{a}^{t}(t-s)^{\alpha-1} h(s) \mathrm{d} s$.

And the following simple calculation can be obtained by (2.4)

$$
\begin{gathered}
c_{k}=-\frac{x_{k}}{k !}, k=0,1,2, \cdots, n-2, \\
c_{n-1}=\frac{x_{b}}{(n-1) !}+\frac{h(a)(b-a)^{\alpha-n+1}}{(n-2) ! \Gamma(\alpha-n+2)}(t-a)^{n-1} \\
-\frac{(t-a)^{n-1}}{(n-1) ! \Gamma(\alpha-n+1)} \int_{a}^{b}(b-s)^{\alpha-n} h(s) \mathrm{d} s .
\end{gathered}
$$

Hence Equation (2.5). Conversely, it is clear that if $x(t)$ satisfies Equation (2.5), then Equations (2.4) hold.

\section{Existence and Uniqueness of Solutions}

In this section, Our first result is based on the Banach fixed point theorem (see [28]).

Theorem 3.1 Assume that

$(H 1)$ There exists a function $\lambda(t) \in C(J, R)$ such that

$$
\begin{aligned}
& |f(t, u(t))-f(t, v(t))| \leq \lambda(t)|u(t)-v(t)|, \\
& \forall t \in J=[a, b] ; u(t), v(t) \in R .
\end{aligned}
$$

If

$$
\theta=I^{\alpha} \lambda(t)+\frac{(b-a)^{\alpha} \lambda(a)}{(n-2) ! \Gamma(\alpha-n+2)}+\frac{(b-a)^{n-1}}{(n-1) !} I^{\alpha-n+1} \lambda(b)<1 .
$$

Then the BVP (1.1) has a unique solution on $J$.

Proof. Transform the problem (1.1) into a fixed point problem. Consider the operator

$$
T: C^{n-1}(J, R) \rightarrow C^{n-1}(J, R)
$$

defined by

$$
\begin{aligned}
\operatorname{Tx}(t)= & \frac{1}{\Gamma(\alpha)} \int_{a}^{t}(t-s)^{\alpha-1} f(s, x(s)) \mathrm{d} s \\
& +\left(\frac{x_{b}}{(n-1) !}+\frac{f(a, x(a))(b-a)^{\alpha-n+1}}{(n-2) ! \Gamma(\alpha-n+2)}\right)(t-a)^{n-1} \\
& -\frac{(t-a)^{n-1}}{(n-1) ! \Gamma(\alpha-n+1)} \int_{a}^{b}(b-s)^{\alpha-n} f(s, x(s)) \mathrm{d} s+\sum_{k=0}^{n-2} \frac{x_{k}}{k !}(t-a)^{k} .
\end{aligned}
$$


The Banach contraction principle is used to prove that $T$ has afixed point.

Let $x(t), y(t) \in C^{n-1}(J, R)$. Then $\forall t \in J$,

$$
\begin{aligned}
|T x(t)-T y(t)| \leq & \frac{1}{\Gamma(\alpha)} \int_{a}^{t}(t-s)^{\alpha-1}|f(s, x(s))-f(s, y(s))| \mathrm{d} s \\
& +\frac{(t-a)^{n-1}(b-a)^{\alpha-n+1}}{(n-2) ! \Gamma(\alpha-n+2)}|f(a, x(a))-f(a, y(a))| \\
& +\frac{(t-a)^{n-1}}{(n-1) ! \Gamma(\alpha-n+1)} \int_{a}^{b}(b-s)^{\alpha-n}|f(s, x(s))-f(s, y(s))| \mathrm{d} s \\
\leq & \frac{\|x-y\|_{\infty}}{\Gamma(\alpha)} \int_{a}^{t}(t-s)^{\alpha-1} \lambda(s) \mathrm{d} s+\frac{(b-a)^{\alpha-n+1} \lambda(a)\|x-y\|_{\infty}}{(n-2) ! \Gamma(\alpha-n+2)} \\
& +\frac{(b-a)^{n-1}\|x-y\|_{\infty}}{(n-1) ! \Gamma(\alpha-n+1)} \int_{a}^{b}(b-s)^{\alpha-n} \lambda(s) \mathrm{d} s \\
= & \left(I^{\alpha} \lambda(t)+\frac{(b-a)^{\alpha} \lambda(a)}{(n-2) ! \Gamma(\alpha-n+2)}+\frac{(b-a)^{n-1}}{(n-1) !} I^{\alpha-n+1} \lambda(b)\right)\|x-y\|_{\infty} .
\end{aligned}
$$

Thus

$$
\|T x-T y\|_{\infty} \leq\left(I^{\alpha} \lambda(t)+\frac{(b-a)^{\alpha} \lambda(a)}{(n-2) ! \Gamma(\alpha-n+2)}+\frac{(b-a)^{n-1}}{(n-1) !} I^{\alpha-n+1} \lambda(b)\right)\|x-y\|_{\infty} .
$$

Consequently, by (3.1) $T$ is a contraction operator. As a consequence of the Banach Fixed point theorem, $T$ has a fixed point which is the unique solution of the problem (1.1). The proof is completed.

In Theorem 3.1, if the function $\lambda(t)$ is replaced by a constant $L>0$, the second result follows.

Theorem 3.2 Assume that

(H2) There exists a constant $L>0($ i.e. $\lambda(t)=L>0)$, such that

$$
\begin{aligned}
& |f(t, u(t))-f(t, v(t))| \leq L|u(t)-v(t)|, \\
& \forall t \in J=[a, b] ; u(t), v(t) \in R .
\end{aligned}
$$

If

$$
\theta=L(b-a)^{\alpha}\left(\frac{1}{\Gamma(\alpha+1)}+\frac{n}{(n-1) ! \Gamma(\alpha-n+2)}\right)<1 .
$$

Then the BVP (1.1) has a unique solution on $J$.

The third result is based on Schauder's Fixed point theorem.

Theorem 3.3 Assume that

(HB) The function $f: J \times R \rightarrow R$ is continuous.

(H4) There exists a constant $M>0$, such that

$$
|f(t, u(t))| \leq M \text { for each } t \in J=[a, b] \text { and } \forall u(t) \in R \text {. }
$$

Then the BVP (1.1) has at least one solution on $J$.

Proof. Schauder's Fixed point theorem is used to prove that $T$ defined by (3.2) has a fixed point. The proof will be given in several steps.

Step 1: $T$ is continuous.

Let $\left\{x_{m}\right\}$ be a sequence such that $x_{m} \rightarrow x$ in $C(J, R)$. Then for each $t \in J$ 


$$
\begin{aligned}
\left|T x_{m}(t)-T x(t)\right| \leq & \frac{1}{\Gamma(\alpha)} \int_{a}^{t}(t-s)^{\alpha-1}\left|f\left(s, x_{m}(s)\right)-f(s, x(s))\right| \mathrm{d} s \\
& +\frac{(t-a)^{n-1}(b-a)^{\alpha-n+1}}{(n-2) ! \Gamma(\alpha-n+2)}\left|f\left(a, x_{m}(a)\right)-f(a, x(a))\right| \\
& +\frac{(t-a)^{n-1}}{(n-1) ! \Gamma(\alpha-n+1)} \int_{a}^{b}(b-s)^{\alpha-n}\left|f\left(s, x_{m}(s)\right)-f(s, x(s))\right| \mathrm{d} s \\
\leq & \frac{1}{\Gamma(\alpha)} \int_{a}^{t}(t-s)^{\alpha-1} \sup _{s \in J}\left|f\left(s, x_{m}(s)\right)-f(s, x(s))\right| \mathrm{d} s \\
& +\frac{(b-a)^{\alpha}}{(n-2) ! \Gamma(\alpha-n+2)} \sup _{s \in J}\left|f\left(s, x_{m}(s)\right)-f(s, x(s))\right| \\
& +\frac{(b-a)^{n-1}}{(n-1) ! \Gamma(\alpha-n+1)} \int_{a}^{b}(b-s)^{\alpha-n} \sup _{s \in J}\left|f\left(s, x_{m}(s)\right)-f(s, x(s))\right| \mathrm{d} s
\end{aligned}
$$

then

$$
\begin{aligned}
& \left|\operatorname{Tx}_{m}(t)-T x(t)\right| \\
& \leq\left\|f\left(\bullet, x_{m}(\bullet)\right)-f(\bullet, x(\bullet))\right\|_{\infty}\left(\frac{1}{\Gamma(\alpha)} \int_{a}^{t}(t-s)^{\alpha-1} \mathrm{~d} s\right. \\
& +\frac{(b-a)^{\alpha}}{(n-2) ! \Gamma(\alpha-n+2)}+\frac{(b-a)^{n-1}}{(n-1) ! \Gamma(\alpha-n+1)} \int_{a}^{b}(b-s)^{\alpha-n} \mathrm{~d} s \\
& \leq\left\|f\left(\bullet, x_{m}(\bullet)\right)-f(\bullet, x(\bullet))\right\|_{\infty}(b-a)^{\alpha}\left(\frac{1}{\Gamma(\alpha+1)}+\frac{n}{(n-1) ! \Gamma(\alpha-n+2)}\right) .
\end{aligned}
$$

Since $f$ is a continuous function, it can be shown that

$$
\left\|T x_{m}-T x\right\|_{\infty} \leq(b-a)^{\alpha}\left(\frac{1}{\Gamma(\alpha+1)}+\frac{n}{(n-1) ! \Gamma(\alpha-n+2)}\right)\left\|f\left(\cdot, x_{m}(\cdot)\right)-f(\cdot, x(\cdot))\right\|_{\infty} .
$$

And hence

$$
\left\|T x_{m}-T x\right\|_{\infty} \rightarrow 0, \quad m \rightarrow \infty .
$$

Step 2: T maps the bounded sets into the bounded sets in $C(J, R)$.

For any $\eta^{*}>0$, it can be shown that there exists a positive constant $\ell$ such that $\forall x \in B_{\eta^{*}}=\left\{x \in C(J, R):\|x\|_{\infty} \leq \eta^{*}\right\},\|T x\|_{\infty} \leq \ell$.

In fact, $\forall t \in J$, by (3.2) and (H4)

$$
\begin{aligned}
|T x(t)| \leq & \sum_{k=0}^{n-2} \frac{\left|x_{k}\right|}{k !}(b-a)^{k}+\left(\frac{\left|x_{b}\right|}{(n-1) !}+\frac{|f(a, x(a))|(b-a)^{\alpha-n+1}}{(n-2) ! \Gamma(\alpha-n+2)}\right)(b-a)^{n-1} \\
& +\frac{1}{\Gamma(\alpha)} \int_{a}^{t}(t-s)^{\alpha-1}|f(s, x(s))| \mathrm{d} s \\
& +\frac{(b-a)^{n-1}}{(n-1) ! \Gamma(\alpha-n+1)} \int_{a}^{b}(b-s)^{\alpha-n}|f(s, x(s))| \mathrm{d} s \\
\leq & \sum_{k=0}^{n-2} \frac{\left|x_{k}\right|}{k !}(b-a)^{k}+\frac{\left|x_{b}\right|(b-a)^{n-1}}{(n-1) !} \\
& +M(b-a)^{\alpha}\left(\frac{1}{\Gamma(\alpha+1)}+\frac{n}{(n-1) ! \Gamma(\alpha-n+2)}\right) .
\end{aligned}
$$


Thus

$$
\|T x\|_{\infty} \leq \ell
$$

where

$$
\begin{aligned}
\ell= & \sum_{k=0}^{n-2} \frac{\left|x_{k}\right|}{k !}(b-a)^{k}+\frac{\left|x_{b}\right|(b-a)^{n-1}}{(n-1) !} \\
& +M(b-a)^{\alpha}\left(\frac{1}{\Gamma(\alpha+1)}+\frac{n}{(n-1) ! \Gamma(\alpha-n+2)}\right) .
\end{aligned}
$$

Step 3: Tmaps the bounded sets into the equicontinuous sets of $C(J, R)$.

Let $t_{1}, t_{2} \in J, t_{1}<t_{2}, B_{\eta^{*}}$ be abounded set of $C(J, R)$ as above, and $x \in B_{\eta^{*}}$

$$
\begin{aligned}
\left|T x\left(t_{2}\right)-T x\left(t_{1}\right)\right| \leq & \mid \frac{1}{\Gamma(\alpha)}\left(\int_{a}^{t_{2}}\left(t_{2}-s\right)^{\alpha-1} f(s, x(s)) \mathrm{d} s-\int_{a}^{t_{1}}\left(t_{1}-s\right)^{\alpha-1} f(s, x(s)) \mathrm{d} s\right) \\
& +\left(\frac{x_{b}}{(n-1) !}+\frac{f(a, x(a))(b-a)^{\alpha-n+1}}{(n-2) ! \Gamma(\alpha-n+2)}\right)\left(\left(t_{2}-a\right)^{n-1}-\left(t_{1}-a\right)^{n-1}\right) \\
& -\frac{\left(t_{2}-a\right)^{n-1}-\left(t_{1}-a\right)^{n-1}}{(n-1) ! \Gamma(\alpha-n+1)} \int_{a}^{b}(b-s)^{\alpha-n} f(s, x(s)) \mathrm{d} s \\
& +\sum_{k=0}^{n-2} \frac{x_{k}}{k !}\left(\left(t_{2}-a\right)^{k}-\left(t_{1}-a\right)^{k}\right) \mid .
\end{aligned}
$$

Then

$$
\begin{aligned}
\left|\operatorname{Tx}\left(t_{2}\right)-\operatorname{Tx}\left(t_{1}\right)\right| \leq & \frac{M}{\Gamma(\alpha)} \int_{a}^{t_{1}}\left(\left(t_{2}-s\right)^{\alpha-1}-\left(t_{1}-s\right)^{\alpha-1}\right) \mathrm{d} s+\frac{M}{\Gamma(\alpha)} \int_{t_{1}}^{t_{2}}\left(t_{2}-s\right)^{\alpha-1} \mathrm{~d} s \\
& +\left(\frac{\left|x_{b}\right|}{(n-1) !}+\frac{M(b-a)^{\alpha-n+1}}{(n-2) ! \Gamma(\alpha-n+2)}\right)\left(\left(t_{2}-a\right)^{n-1}-\left(t_{1}-a\right)^{n-1}\right) \\
& +\frac{M\left(\left(t_{2}-a\right)^{n-1}-\left(t_{1}-a\right)^{n-1}\right)}{(n-1) ! \Gamma(\alpha-n+1)} \int_{a}^{b}(b-s)^{\alpha-n} \mathrm{~d} s \\
& +\sum_{k=0}^{n-2} \frac{\left|x_{k}\right|}{k !}\left(\left(t_{2}-a\right)^{k}-\left(t_{1}-a\right)^{k}\right) \\
\leq & \frac{M}{\Gamma(\alpha+1)}\left(\left(t_{2}-a\right)^{\alpha}-\left(t_{1}-a\right)^{\alpha}\right)+\sum_{k=0}^{n-2} \frac{\left|x_{k}\right|}{k !}\left(\left(t_{2}-a\right)^{k}-\left(t_{1}-a\right)^{k}\right) \\
& +\left(\frac{\left|x_{b}\right|}{(n-1) !}+\frac{n M(b-a)^{\alpha-n+1}}{(n-1) ! \Gamma(\alpha-n+2)}\right)\left(\left(t_{2}-a\right)^{n-1}-\left(t_{1}-a\right)^{n-1}\right) .
\end{aligned}
$$

As $t_{1} \rightarrow t_{2}$, the right-hand side of the aboveinequality tends to zero. As a consequence of Steps 1 to 3 together with the Arzelá-Ascoli theorem, $T: C(J, R) \rightarrow C(J, R) \quad$ is completely continuous.

Step 4: A priori bounds.

Let $\varepsilon=\{x \in C(J, R): x=\lambda T x$ for some $0<\lambda<1\}$, it shall be shown that 
the set is bounded.

Let $x \in \varepsilon$, then $x=\lambda T x$ for some $0<\lambda<1$. Thus $\forall t \in J$,

$$
\begin{aligned}
x= & \lambda T x \\
= & \frac{\lambda}{\Gamma(\alpha)} \int_{a}^{t}(t-s)^{\alpha-1} f(s, x(s)) \mathrm{d} s \\
& +\lambda\left(\frac{x_{b}}{(n-1) !}+\frac{f(a, x(a))(b-a)^{\alpha-n+1}}{(n-2) ! \Gamma(\alpha-n+2)}\right)(t-a)^{n-1} \\
& -\frac{\lambda(t-a)^{n-1}}{(n-1) ! \Gamma(\alpha-n+1)} \int_{a}^{b}(b-s)^{\alpha-n} f(s, x(s)) \mathrm{d} s+\lambda \sum_{k=0}^{n-2} \frac{x_{k}}{k !}(t-a)^{k} .
\end{aligned}
$$

By the condition (H4) and Step 2,

$$
\begin{aligned}
|x(t)| \leq & \sum_{k=0}^{n-2} \frac{\left|x_{k}\right|}{k !}(b-a)^{k}+\frac{\left|x_{b}\right|(b-a)^{n-1}}{(n-1) !} \\
& +M(b-a)^{\alpha}\left(\frac{1}{\Gamma(\alpha+1)}+\frac{n}{(n-1) ! \Gamma(\alpha-n+2)}\right) .
\end{aligned}
$$

Thus for every $\forall t \in J$,

$$
\begin{aligned}
\|x\|_{\infty} \leq & \sum_{k=0}^{n-2} \frac{\left|x_{k}\right|}{k !}(b-a)^{k}+\frac{\left|x_{b}\right|(b-a)^{n-1}}{(n-1) !} \\
& +M(b-a)^{\alpha}\left(\frac{1}{\Gamma(\alpha+1)}+\frac{n}{(n-1) ! \Gamma(\alpha-n+2)}\right):=R .
\end{aligned}
$$

This shows that the set $\varepsilon$ is bounded. As a consequence of Schauder's fixed point theorem, Thas a fixed point which is a solution of the problem (1.1).

In Theorem 3.3, if the condition (H4) is weakened, the fourth result can be obtained, which is a more general existence result (see [6]).

Theorem 3.4 Assume that $(H B)$ and the following conditionshold.

(H5) There exist a functional $\psi_{f} \in L^{1}\left(J, R^{+}\right)$and a continuous and nondecreasing $\varphi:[0, \infty) \rightarrow(0, \infty)$, such that

$$
|f(t, x(t))| \leq \psi_{f}(t) \varphi(|x(t)|)
$$

for each $t \in J=[a, b]$ and $\forall x(t) \in R$.

(H6) There exists a number $K>0$, such that

$$
\begin{aligned}
\theta= & K^{-1}\left(\varphi(K)\left\|I^{\alpha} \psi_{f}\right\|_{L^{1}}+\frac{\lambda(b-a)^{n-1} \varphi(K)}{(n-1) !} I^{\alpha-n+1} \psi_{f}(b)+\sum_{k=0}^{n-2} \frac{\left|x_{k}\right|}{k !}(b-a)^{k}\right. \\
& \left.+\frac{\left|x_{b}\right|}{(n-1) !}(b-a)^{n-1}+\frac{\psi_{f}(a) \varphi(\mid x((a) \mid)}{(n-2) ! \Gamma(\alpha-n+2)}(b-a)^{\alpha}\right)<1 .
\end{aligned}
$$

Then the BVP (1.1) has at least one solution on $J$.

Proof. Consider the operator $T$ defined by (3.2), $\forall \lambda \in[0,1], t \in J=[a, b]$, let 
$x(t)$ meets $x(t)=\lambda(T x)(t)$, then from $(H 5)$ and $(H 6)$,

$$
\begin{aligned}
|x(t)|= & |\lambda(T x)(t)| \leq|T x(t)| \leq \frac{1}{\Gamma(\alpha)} \int_{a}^{t}(t-s)^{\alpha-1} f(s, x(s)) \mathrm{d} s \\
& +\left|\left(\frac{x_{b}}{(n-1) !}+\frac{f(a, x(a))(b-a)^{\alpha-n+1}}{(n-2) ! \Gamma(\alpha-n+2)}\right)(t-a)^{n-1}\right| \\
& +\frac{\left|(t-a)^{n-1}\right|}{(n-1) ! \Gamma(\alpha-n+1)} \int_{a}^{b}(b-s)^{\alpha-n}|f(s, x(s))| \mathrm{d} s+\sum_{k=0}^{n-2} \frac{\left|x_{k}\right|}{k !}(t-a)^{k} \\
\leq & \frac{\varphi\left(\|x\|_{\infty}\right)}{\Gamma(\alpha)} \int_{a}^{t}(t-s)^{\alpha-1} \psi_{f}(s) \mathrm{d} s \\
& +\frac{\left|x_{b}\right|}{(n-1) !}(b-a)^{n-1}+\frac{\psi_{f}(a) \varphi(|x(a)|)(b-a)^{\alpha}}{(n-2) ! \Gamma(\alpha-n+2)} \\
& +\frac{\varphi\left(\|x\|_{\infty}\right)(b-a)^{n-1}}{(n-1) ! \Gamma(\alpha-n+1)} \int_{a}^{b}(b-s)^{\alpha-n} \psi_{f}(s) \mathrm{d} s+\sum_{k=0}^{n-2} \frac{\left|x_{k}\right|}{k !}(b-a)^{k} \\
\leq & \varphi\left(\|x\|_{\infty}\right) \mid I^{\alpha} \psi_{f} \|_{L^{1}}+\frac{\left|x_{b}\right|}{(n-1) !}(b-a)^{n-1}+\frac{\psi_{f}(a) \varphi(|x(a)|)(b-a)^{\alpha}}{(n-2) ! \Gamma(\alpha-n+2)} \\
& +\frac{\varphi\left(\|x\|_{\infty}\right)(b-a)^{n-1}}{(n-1) !} I^{\alpha-n+1} \psi_{f}(b)+\sum_{k=0}^{n-2} \frac{\left|x_{k}\right|}{k !}(b-a)^{k} .
\end{aligned}
$$

By (H6), there exists $K$ such that $\|x\|_{\infty} \neq K$. Let $D=\left\{x \in C(J, R):\|x\|_{\infty}<K\right\}$, the operator $T: \bar{D} \rightarrow C(J, R)$ is completely continuous. Through proper selection of $D$, there exists no $x(t) \in \partial D$ such that $x(t)=\lambda(T x)(t)$ for some $\lambda \in(0,1)$.

Therefore, $T$ is Leray-Schauder type operator (see [6]), so that it has a fixed point $x(t)$ in $\bar{U}$, which is a solution of the BVP (1.1).

\section{An Example}

For the boundary value problem

$$
\begin{cases}{ }^{c} D_{a}^{\alpha} x(t)=\frac{|x(t)| t}{1+x(t)}, \quad t \in J=[0,1], & n-1<\alpha \leq n, \\ x^{(\mathrm{k})}(0)=0, \quad k=0,1,2, \cdots, n-2 ; \quad x^{(n-1)}(1)=1 .\end{cases}
$$

Take

$$
f(t, u(t))=\frac{u(t) t}{1+u(t)},(t, u(t)) \in J \times[0, \infty) .
$$

Let $x(t), y(t) \in[0, \infty), t \in J$. Then

$$
\begin{aligned}
|f(t, x(t))-f(t, y(t))| & =t\left|\frac{x(t)}{1+x(t)}-\frac{y(t)}{1+y(t)}\right| \\
& =\frac{t|x(t)-y(t)|}{(1+x(t))(1+y(t))} \\
& \leq t|x(t)-y(t)| .
\end{aligned}
$$


Hence the condition $(H 1)$ holds with $\lambda(t)=t \in C(J, R)$. It can be checked that condition (3.2) is satisfied with $b=1$. In fact,

$$
\begin{aligned}
\theta & =I^{\alpha} \lambda(t)+\frac{\lambda(0)}{(n-2) ! \Gamma(\alpha-n+2)}+\frac{1}{(n-1) !} I^{\alpha-n+1} \lambda(1) \\
& =\frac{1}{\Gamma(\alpha+2)} t^{\alpha+1}+\frac{1}{(n-1) ! \Gamma(\alpha-n+3)}<1, \quad(t \leq 1, \lambda(0)=0)
\end{aligned}
$$

only if

$$
\frac{1}{\Gamma(\alpha+2)}+\frac{1}{(n-1) ! \Gamma(\alpha-n+3)}<1
$$

For example, $\alpha=\frac{5}{2}$, then $n=[\alpha]+1=3, \quad \Gamma(\alpha+2)=\Gamma\left(\frac{9}{2}\right)=\frac{105 \sqrt{\pi}}{16}$,

$$
\begin{aligned}
\Gamma(\alpha-n+3)=\Gamma(\alpha)= & \Gamma\left(\frac{5}{2}\right)=\frac{3 \sqrt{\pi}}{4},(n-1) !=2 !=2 . \text { Then } \\
\theta & \leq \frac{1}{\Gamma(\alpha+2)}+\frac{1}{(n-1) ! \Gamma(\alpha-n+3)} \\
& =\frac{1}{\Gamma(\alpha+2)}+\frac{1}{2 \Gamma(\alpha)}=\left(\frac{16}{105}+\frac{2}{3}\right) \frac{1}{\sqrt{\pi}} \\
& =0.4621<1 .
\end{aligned}
$$

Then by Theorem 3.1 the boundary value problem (4.1) has a uniquesolution on $J=[0,1]$ for the values of $\alpha \in(2,3]$.

\section{Acknowledgements}

The authors would like to thank the reviewers for their valuable suggestions and comments, which improved the completeness of the paper. Research of J. Song is funded by the High-level Talents Project of Guangdong Province Colleges and Universities (2013-178). Research of Z. Zeng is funded by the Natural Science Foundation of Guangdong Province of China (S2012010010069). These supports are greatly appreciated.

\section{References}

[1] Oldham, K.B. and Spanier, J. (1974) The Fractional Calculus. Academic Press, New York, London.

[2] Miller, K.S. and Ross, B. (1993) An Introduction to the Fractional Calculus and Fractional Differential Equations. John Wiley \& Sons, Inc., New York/Singapore.

[3] Podlubny, I. (1999) Fractional Differential Equations. Academic Press, New York/ London.

[4] He, J.H. (1999) Some Applications of Nonlinear Fractional Differential Equations and Their Approximations. Bulletin of Science, Technology \& Society, 15, 86-90.

[5] Hilfer, R. (2000) Applications of Fractional Calculus in Physics. World Scientific, Singapore. https://doi.org/10.1142/3779

[6] Agarwal, R.P., Benchohra, M. and Hamani, S. (2009) Boundary Value Problems for Fractional Differential Equations. Georgian Mathematical Journal, 16, 401-411.

[7] El-Sayed, A.M.A. (1988) Fractional Differential Equations. Kyungpook Mathemati- 
cal Journal, 28, 119-122.

[8] El-Sayed, A.M.A. and Ibrahim, A.G. (1995) Multivalued Fractional Differential Equations. Applied Mathematics and Computation, 68, 15-25.

[9] El-Sayed, A.M.A. (1998) Nonlinear Functional Differential Equations of Arbitrary Orders. Nonlinear Analysis, 33, 181-186.

[10] El-Sayed, A.M.A. and El-Salam, S.A.A. (2009) Nonlocal Boundary Value Problem of a Fractional-Order Functional Differential Equation. International Journal of Nonlinear Science, 7, 436-442.

[11] Benchohra, M., Hamani, S. and Ntouyas, S.K. (2008) Boundary Value Problems for Differential Equations with Fractional Order. Surveys in Mathematics and Its Applications, 3, 1-12.

[12] Benchohra, M., Hamani, S. and Ntouyas, S.K. (2009) Boundary Value Problems for Differential Equations with Fractional Order and Nonlocal Conditions. Nonlinear Analysis, 71, 2391-2396.

[13] Yu, C. and Gao, G. (2005) Existence of Fractional Differential Equations. Journal of Mathematical Analysis and Applications, 310, 26-29.

[14] Yu, C. and Gao, G. (2005) On the Solution of Nonlinear Fractional Order Differential Equation. Nonlinear Analysis, 63, e971-e976.

[15] Zhang, S.Q. (2006) Positive Solutions for Boundary-Value Problems of Nonlinear Fractional Differential Equations. Electronic Journal of Differential Equations, 36, 1-12. https://doi.org/10.1155/ADE/2006/90479

[16] Diethelm, K. (2010) The Analysis of Fractional Differential Equations. SpringerVerlag, Berlin. https://doi.org/10.1007/978-3-642-14574-2

[17] Sun, Y.F. and Wang, P.G. (2010) Iterative Methods for a Fourth-Order Differential Equations with Retardation and Anticipation. Dynamics of Continuous, Discrete and Impulsive Systems, Series B: Applications \& Algorithms, 17, 487-500.

[18] Sun, Y.F. and Wang, P.G. (2010) Quasilinear Iterative Scheme for a Fourth-Order Differential Equation with Retardation and Anticipation. Applied Mathematics and Computation, 217, 3442-3452.

[19] Agarwal, R.P., Zhou, Y. and He, Y.Y. (2010) Existence of Fractional Neutral Functional Differential Equations. Computers \& Mathematics with Applications, 59, 1095-1100.

[20] Nyamoradi, N. (2012) Existence of Solutions for Multi Point Boundary Value Problems for Fractional Differential Equations. Arab Journal of Mathematical Sciences, 18, 165-175.

[21] Sakthivel, R., Revathi, P. and Ren, Y. (2013) Existence of Solutions for Nonlinear Fractional Stochastic Differential Equations. Nonlinear Analysis. Theory, Methods \& Applications, 81, 70-86.

[22] Deng, J.W., Zhao, L.J. and Wu, Y.J. (2015) Efficient Algorithms for Solving the Fractional Ordinary Differential Equations. Applied Mathematics and Computation, 269, 196-216.

[23] Brzdek, J. and Eghbali, N. (2016) On Approximate Solutions of Some Delayed Fractional Differential Equations. Applied Mathematics Letters, 54, 31-35.

[24] Lakshmikantham, V. and Vatsala, A.S. (2008) General Uniqueness and Monotone Iterative Technique for Fractional Differential Equations. Applied Mathematics Letters, 21, 828-834.

[25] Lakshmikantham, V. and Vatsala, A.S. (2008) Basic Theory of Fractional Differential Equations. Nonlinear Analysis, 69, 2677-2682. 
[26] Lakshmikantham, V. and Devi, J.V. (2008) Theory of Fractional Differential Equations in a Banach Space. European Journal of Pure and Applied Mathematics, 1, $38-45$.

[27] Samko, S.G., Kilbas, A.A. and Marichev, O.I. (1993) Fractional Integral and Derivative, Theory and Applications. Gordon and Breach Science Publishers, Philadelphia.

[28] Agarwal, R.P., Meehan, M. and O’Regan, D. (2008) Fixed Point Theory and Applications. Cambridge University Press \& Beijing World Publishing Corporation.

\section{Scientific Research Publishing}

Submit or recommend next manuscript to SCIRP and we will provide best service for you:

Accepting pre-submission inquiries through Email, Facebook, LinkedIn, Twitter, etc. A wide selection of journals (inclusive of 9 subjects, more than 200 journals)

Providing 24-hour high-quality service

User-friendly online submission system

Fair and swift peer-review system

Efficient typesetting and proofreading procedure

Display of the result of downloads and visits, as well as the number of cited articles

Maximum dissemination of your research work

Submit your manuscript at: http://papersubmission.scirp.org/

Or contact am@scirp.org 